GRADUATE THESIS REPORT

\title{
Use of mobile technologies in the treatment of alcohol use disorder
}

\author{
Leonardo J. Gutiérrez, Luis A. Castro, Oresti Banos
}

Published: 30 November 2021

\begin{abstract}
Mental disorders currently affect more than 500 million people in the world, among which, substance use disorders, such as alcohol, represent a major social problem, especially in developing countries. The use of mobile technologies in combination with behavioral and intervention sampling methodologies such as EMA and EMI allow a collection of information and intervention in realtime and in natural environments, which is of great benefit when dealing with patients suffering from mental disorders both in diagnosis and treatment. This work shows the stages of research, design, and development of experiments of a 4-year doctoral work, which aims to identify challenges at the intersection between mobile technologies and alcohol use disorder and propose different solutions to them.
\end{abstract}

\section{Keywords:}

Mobile sensing; EMA; EMI; Mental health; Substance use disorder; Alcohol use disorder.

\section{Introducción}

Un trastorno mental se define como un síndrome o patrón conductual o psicológico que ocurre en un individuo, de los que actualmente más de 500 millones de personas sufren de ellos [1]. Los trastornos por el uso de sustancias generalmente implican patrones de comportamiento en los que las personas continúan consumiendo una sustancia a pesar de experimentar problemas derivados de ello [2]. El trastorno por consumo de sustancias generalmente se da en comorbilidad con otros trastornos y enfermedades. Por ejemplo, el trastorno por consumo de alcohol suele presentarse en comorbilidad con trastornos como depresión o trastorno bipolar al estar intoxicado o en la abstinencia [2].

El trastorno por consumo de alcohol es un patrón de ingesta de alcohol descontrolada. El trastorno por consumo de alcohol puede ser leve, moderado o grave, según la cantidad de síntomas que se presenten [2]. Instrumentos como el AUDIT (Alcohol Use

Gutierrez, Leonardo J., Castro, Luis A.

Instituto Tecnológico de Sonora (ITSON)

Ciudad Obregón, Sonora, México.

leonardo.gutierrez@potros.itson.edu.mx, luis.castro@acm.org

Banos, Oresti.

Universidad de Granada

Granada, España.

oresti@ugr.es
Disorders Identification Test) se utilizan en la práctica clínica para determinar si el consumo de alcohol de una persona puede considerarse peligroso [3].

Algunos de los métodos para el estudio del comportamiento con los EMAs (Ecological Momentary Assessment) o ESMs (Experience Sampling Method), las cuales son técnicas de muestreo de comportamientos y experiencias de los sujetos en tiempo real, en sus entornos naturales [5]. Por otra parte, los EMIs (Ecological Momentary Intervention) son tratamientos que se brindan a las personas durante su vida cotidiana (en tiempo) y en entornos naturales (mundo real) mediante retroalimentación, siendo esta la forma en que se presenta la intervención con el paciente en tiempo real en forma de mensajes motivacionales o de auto-ayuda, retroalimentación semi-individualizada y uso de gráficos [4]. Por ejemplo, un mensaje puede ser "¡Lamento oír que no se siente bien! ¡La próxima vez marque el ritmo de sus bebidas y asegúrese de comer lo suficiente antes de que comience la noche!" refiriéndose a la cantidad y forma en la que se consumieron bebidas alcohólicas una noche anterior.

Por último, la combinación de estas técnicas de muestreo e intervención oportunista (EMA y EMI) con tecnologías de sensado móvil abren las puertas a nuevas direcciones de seguimiento y tratamiento con pacientes que padecen trastornos de comportamiento. En particular, aquellos individuos con problemas de consumo de alcohol, los cuales pueden requerir de intervención al momento, esto debido a los síntomas y situaciones que se pueden presentar durante un síndrome de abstinencia o intoxicación.

\section{Sensado móvil en el trastorno por consumo de alcohol}

En la intersección entre las tecnologías de sensado móvil y el trastorno por consumo de alcohol se ha realizado distintos tipos de trabajos. Un ejemplo de ellos es la utilización de relojes inteligentes como es el caso de WatchOver, en el cual utilizaron un reloj inteligente para predecir patrones de conducta de consumo de sustancias, como el alcohol y la marihuana. De manera adicional, se ofrecía intervención en tiempo real mediante micro intervenciones (micro-EMI's), las cuales se refieren a intervenciones breves con duración de segundos [6].

Por otra parte, los teléfonos inteligentes (smartphones en inglés) suelen ser utilizados como herramienta de soporte en el tratamiento de pacientes que padecen de trastornos por consumo de sustancias Un ejemplo es Soberdiary, el cual es un sistema de soporte para la recuperación de pacientes con problemas de consumo de alcohol basado en el uso de teléfonos inteligentes, permitiendo la auto-monitorización de las conductas de consumo alcohol [7]. 
Por último, se han desarrollado modelos de aprendizaje máquina para la detección de niveles de alcohol en la sangre utilizando teléfonos inteligentes, los autores en [8] desarrollaron un modelo que permitía identificar niveles de alcohol en la sangre de los participantes mediante la realización de distintas tareas simples en el smartphone.

Existe poca investigación en el cruce de EMIs, sensado móvil y los trastornos por consumo de alcohol, anteriormente se han mencionado algunos de estos ejemplos, como es el uso de dispositivos inteligentes, como smartphones y smartwatch, para ayudar en el seguimiento y tratamiento de pacientes que padecen de este trastorno. Esto lleva a plantear las siguientes preguntas de investigación.

\section{Preguntas de investigación}

En mi trabajo de doctorado se plantea responder estas dos preguntas de investigación principales:

- ¿Qué efecto tienen los EMIs en los patrones de consumo de sustancias al utilizar una plataforma de sensado móvil para dar apoyo al tratamiento en personas con problemas de ingesta de sustancias?

- ¿Cuáles son los efectos de los distintos tipos de EMIs en los patrones de consumo de sustancias?

Para resolver estas preguntas decidimos iniciar con la realización de un estudio de aceptación tecnológica, el cual nos permitiría tener un entendimiento de las actitudes y tendencias de uso de tecnologías móviles por parte de los usuarios finales.

\section{Metodología}

En esta sección se muestra la metodología a desarrollar durante el trabajo doctoral a 4 años, los cuales se presentan por año:

- Año 1: Explorando el problema. Consiste en un análisis de la literatura sobre la intersección del sensado móvil y los trastornos mentales. Además, incluye un estudio cualitativo con psicólogos, psiquiatras y neuropsicólogos con la finalidad de entender la relación entre apreciaciones sintomatológicas y el contexto del paciente.

- Año 2: Diseño de herramienta de intervenciones móviles. Consiste en un estudio de para conocer las actitudes, intenciones y motivaciones de uso de aplicaciones de sensado móvil para la identificación de patrones de consumo de sustancias basado en el uso del modelo de aceptación tecnológica (TAM). Además, incluye sesiones de diseño con profesionales al cuidado de pacientes en recuperación por problemas del consumo de alcohol y otras drogas.

- Año 3: Desarrollo de herramienta de intervenciones móviles. Incluye el desarrollo de tecnología de sensado móvil de intervención mediante EMIs. Posteriormente, un estudio de campo con personas diagnosticadas con trastorno de abuso de sustancias con la finalidad de evaluar el efecto que tienen los distintos tipos de EMIs en los patrones de consumo de alcohol de los participantes.

- Año 4: Evaluación de herramienta de intervenciones móviles. Incluye una evaluación sumativa de la tecnología de sensado móvil de intervención mediante EMIs.

\section{Avances hasta el momento}

En esta sección se presentará la actualidad del trabajo doctoral, el cual se encuentra en la segunda mitad del 2do año de trabajo, que comprende de un estudio de aceptación tecnológica como se muestra a continuación.

\section{$5.1 \quad$ Año 1: Resultados}

Se realizó un estudio cualitativo mediante 10 entrevistas semiestructuradas con profesionales de la salud (seis mujeres) con experiencia clínica con pacientes con trastornos mentales y / o conductuales, de los cuales 8 eran psicólogos, 1 psiquiatra y 1 neuropsicólogo. Para la entrevista, pedimos a los profesionales de la salud que recordaran un caso particular (es decir, un paciente) que consideran relevante para nuestra investigación.

El protocolo de entrevista incluyó 32 preguntas abiertas relacionadas con el diagnóstico y el tratamiento, e incluyó preguntas sobre el uso de herramientas e instrumentos para apoyar los criterios de diagnóstico. También les hicimos preguntas relacionadas con el uso de la tecnología en su práctica profesional. Todos los participantes firmaron un consentimiento informado después de explicar el propósito del estudio.

De nuestro análisis, obtuvimos tres (3) temas que eran de interés para los profesionales de la salud, como son los pensamientos, sentimientos y comportamientos de los pacientes fuera del consultorio del profesional de la salud, el progreso de los pacientes a lo largo del tiempo y las tecnologías para respaldar los servicios de salud mental.

Posteriormente, identificamos retos a partir de la información provista por los profesionales de la salud referentes a los temas presentados en nuestro análisis. Primero, consideramos que las tecnologías móviles deben realizar inferencias sobre los pensamientos, sentimientos y comportamientos del paciente fuera del consultorio del médico. Por ejemplo, los pacientes que padecen trastornos depresivos mayores pueden tener pensamientos suicidas cuando están solos, lo que puede conducir a una posible muerte. Además, los pacientes que padecen trastornos de ansiedad tienden a evitar las situaciones sociales como mecanismo de afrontamiento, lo que puede repercutir negativamente en su tratamiento. Por el contrario, los pacientes que padecen un trastorno por abuso de sustancias utilizan su entorno social para disfrutar de su consumo de sustancias, algo que nuestros especialistas mencionaron es pertinente detectar y evaluar de antemano.

En segundo lugar, la percepción de los pacientes sobre su progreso a través del tiempo puede cambiar debido a la enfermedad, los efectos secundarios del tratamiento u otros eventos, que, como lo presentaron nuestros participantes, parece tener un efecto importante en la forma en que sus pacientes responden al tratamiento. Por ejemplo, nuestros participantes están realmente interesados en cómo se presentan los pacientes cuando van a la consulta, específicamente en la forma en que hablan, cómo se visten o si hay algún cambio significativo en términos de físico corporal. Un ejemplo es que los pacientes con trastorno límite de la personalidad pueden sufrir cambios bruscos de humor de momento a momento que conducen a cambios drásticos en la autoevaluación o la percepción de la realidad, lo que puede interferir con la forma en que se ven a sí mismos, reduciendo en última instancia la interacción entre el paciente y la tecnología.

En tercer lugar, el uso de tecnologías para apoyar la práctica clínica había polarizado las opiniones de nuestros participantes. Algunos prevén beneficios, como el uso de aplicaciones de mensajería para proporcionar una forma rápida y continua de comunicación médico-paciente y la posibilidad de interacción médico-médico al discutir ciertos casos, mientras que otros temen perder la interacción médico-paciente, argumentando que es parte fundamental del tratamiento. Además, al diseñar y desarrollar dispositivos móviles para apoyar la práctica clínica, es importante considerar la heterogeneidad entre los trastornos, ya sea que los pacientes sufran el mismo trastorno o diferentes. Por ejemplo, 
nuestros participantes mencionaron que, aunque dos pacientes pueden sufrir el mismo trastorno, los síntomas, el seguimiento y la terapia pueden diferir significativamente.

\subsection{Año 2: Avances}

El modelo de aceptación de tecnología es una forma de modelar la adopción potencial de software o hardware. Esto permite que un equipo identifique desafíos potenciales y desarrolle soluciones para superar las dudas de los usuarios. El uso real del sistema es el punto final donde las personas usan la tecnología.

Se reclutarán 20 participantes de centros regionales de atención para personas con problemas de consumo de sustancias. Los participantes deberán ser personas previamente diagnosticadas con trastorno por consumo de alcohol.

Los materiales por utilizar en el estudio son los siguientes:

- Modelo de aceptación tecnológica (TAM) [9]

- Presentación de escenarios de situaciones de riesgo de consumo de sustancias y de uso de tecnologías.

- Cuestionario de evaluación del Modelo de Aceptación Tecnológica (TAM)

Se les mostrará a los participantes un video referente a las situaciones de riesgo de consumo de alcohol. Posteriormente, se les mostrará un escenario de uso de la tecnología para la identificación de patrones de consumo de alcohol. Al finalizar se les solicitará a las personas que respondan una encuesta referente a la utilidad percibida y la facilidad de uso percibida de la tecnología. Finalmente se realizará un análisis de los resultados para identificar la facilidad de uso y la utilidad percibida de la tecnología para la identificación de patrones de consumo de alcohol.

\subsubsection{Resultados esperados}

Se espera obtener el grado de facilidad de uso y de utilidad percibida de tecnologías de sensado móvil para la identificación de patrones de consumo de alcohol basado en el modelo de aceptación tecnológica (TAM).

Para esto es importante conocer las capacidades de uso tecnológico de los usuarios que participaran en el estudio, así como las variables externas que puedan facilitar o dificultar el tiempo y la forma que tomará a los usuarios el aprender a utilizar nuestra aplicación.

Posteriormente, es importante conocer el grado de utilidad percibida por parte de los usuarios con relación a las tecnologías móviles, la cual, en caso de ser positiva, puede derivar en una mayor intención de uso de dichas tecnologías. Por otra parte, en caso de ser negativa, nos permitirá identificar aquellos retos y problemáticas a solucionar antes de iniciar con el desarrollo de la tecnología.

\section{Contribuciones esperadas}

En este trabajo doctoral, específicamente en el año 1, se identificaron retos y limitaciones en la intersección de las áreas de sensado móvil y trastornos mentales, con la finalidad de que presenten las bases para trabajos futuros. Por otra parte, se identificaron situaciones y características contextuales de consumo en pacientes que padecen de trastornos por consumo de alcohol, con la finalidad de proveer un muestreo e intervención individualizada según las necesidades de cada paciente haciendo uso de tecnologías móviles.

Por otra parte, en el año 2 pretendemos realizar un estudio de aceptación tecnológica para conocer las actitudes, intenciones y motivaciones de uso de aplicaciones de sensado móvil para la identificación de patrones de consumo de alcohol basado en el uso del modelo de aceptación tecnológica (TAM). Además, incluye sesiones de diseño con profesionistas al cuidado de pacientes en recuperación por problemas del consumo de alcohol y otras drogas.

Continuando con el año 3, esperamos mostrar los efectos de los distintos tipos de EMIs en los patrones de consumo de alcohol mediante una herramienta de intervenciones móviles, esto refiriéndose a los tipos de mensajes que se suelen utilizar en las intervenciones en tiempo real, esperando el presentar información que pueda ser de ayuda para el desarrollo futuro de sistemas de muestreo e intervención oportunista tanto en personas que padecen trastornos por consumo de alcohol, como en aquellas que padecen otros trastornos por consumo de sustancias y/o trastornos mentales o de comportamiento.

Por último, en el año 4, culminaremos con una evaluación de los resultados obtenidos por la herramienta de intervenciones móviles, utilizando metodologías de análisis probadas en otros estudios con la finalidad de obtener resultados fiables, que puedan ser utilizados y/o comparados en otras investigaciones.

\section{Retos}

Durante el desarrollo de nuestra investigación hemos encontrado retos relacionados con la forma en la que se debe proveer la información al usuario, la cual debe ser en función de las características del trastorno y la persona a tratar. De igual forma, identificamos la necesidad de individualizar las intervenciones con pacientes entorno a características contextuales del mismo, como el sexo, edad, situación social, etc. Además, consideramos que es necesario el considerar el momento más adecuado para la valoración e intervención en los pacientes, esto debido a que la mayoría de las intervenciones y valoraciones suelen ser en tiempos pre-establecidos, lo cual puede traer distintas problemáticas dependiendo del contexto de cada paciente.

Por otra parte, los pacientes suelen llenar auto-reportes acerca de su día a día, lo cual, al tratar con pacientes que padecen de trastornos mentales, específicamente el abuso de sustancias, provee con un gran reto ya que la naturaleza de las sustancias y los efectos que estas causan pueden provocar cambios repentinos de comportamiento y percepción de la realidad en los pacientes.

Por último, en cuanto a los retos como estudiante de doctorado me gustaría obtener retroalimentación de parte de los expertos en cuanto a la mejor metodología para analizar los efectos que tienen los distintos tipos de EMIs en los patrones de consumo de alcohol de los pacientes, además de conocer posibles experiencias que hayan tenido trabajando en esta área o en alguna similar. Por otra parte, me gustaría conocer la opinión de los panelistas acerca del uso de EMAs y EMIs para la valoración y tratamiento de trastornos mentales en general, cuál es su expectativa y posibles ideas de trabajo a futuro.

\section{Agradecimientos}

Se agradece al Consejo Nacional de Ciencia y Tecnología (CONACYT) en México por la beca proporcionada al primer autor.

\section{Referencias}

[1] M. Grigg and S. Saxena, "Promoting mental health nursing research in low and middle income countries," International Nursing Review, vol. 51, no. 4. pp. 194-195, 2004. doi: 10.1111/j.1466-7657.2004.00268.x.

[2] American Psychiatric Association. (2013). Substance use disorders. In Diagnostic and statistical manual of mental disorders (5th ed.).

[3] Babor, T. F., Higgins-Biddle, J. C., Saunders, J. B., \& Monteiro, M. G. (2001). Audit. The Alcohol Use Disorders 
Identification Test (AUDIT): guidelines for use in primary care.

[4] Heron, K. E., \& Smyth, J. M. (2010). Ecological momentary interventions: incorporating mobile technology into psychosocial and health behaviour treatments. British journal of health psychology, 15(1), 1-39.

[5] Shiffman, S., Stone, A. A., \& Hufford, M. R. (2008). Ecological momentary assessment. Annu. Rev. Clin. Psychol., 4, 1-32.

[6] Kunchay, S., \& Abdullah, S. (2020, September). WatchOver: using Apple watches to assess and predict substance co-use in young adults. In Adjunct Proceedings of the 2020 ACM International Joint Conference on Pervasive and Ubiquitous Computing and Proceedings of the 2020 ACM International Symposium on Wearable Computers (pp. 488-493).
[7] Wang, K. C., Hsieh, Y. H., Yen, C. H., You, C. W., Chen, Y. C., Huang, M. C., ... \& Chu, H. H. (2014, September). Soberdiary: A phone-based support system for assisting recovery from alcohol dependence. In Proceedings of the 2014 ACM International Joint Conference on Pervasive and Ubiquitous Computing: Adjunct Publication (pp. 311-314).

[8] Mariakakis, A., Parsi, S., Patel, S. N., \& Wobbrock, J. O. (2018, April). Drunk user interfaces: Determining blood alcohol level through everyday smartphone tasks. In Proceedings of the 2018 CHI Conference on Human Factors in Computing Systems (pp. 1-13).

[9] Davis, F. D. (1989). Perceived usefulness, perceived ease of use, and user acceptance of information technology. MIS quarterly, 319-340.

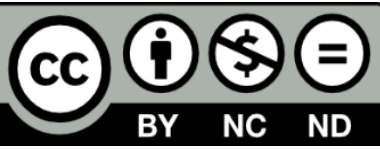

(C) 2021 by the authors. This work is licensed under the Creative Commons AttributionNonCommercial-NoDerivatives 4.0 International License. To view a copy of this license, visit http://creativecommons.org/licenses/by-nc-nd/4.0/ or send a letter to Creative Commons, PO Box 1866, Mountain View, CA 94042, USA. 